\title{
HOLONOMY ON POISSON MANIFOLDS AND THE MODULAR CLASS
}

\author{
VIKTOR L. GINZBURG AND ALEX GOLUBEV
}

\begin{abstract}
We introduce linear holonomy on Poisson manifolds. The linear holonomy of a Poisson structure generalizes the linearized holonomy on a regular symplectic foliation. However, for singular Poisson structures the linear holonomy is defined for the lifts of tangential path to the cotangent bundle (cotangent paths). The linear holonomy is closely related to the modular class studied by A. Weinstein. Namely, the logarithm of the determinant of the linear holonomy is equal to the integral of the modular vector field along such a lift. This assertion relies on the notion of the integral of a vector field along a cotangent path on a Poisson manifold, which is also introduced in the paper.

In the second part of the paper we prove that for locally unimodular Poisson manifolds the modular class is an invariant of Morita equivalence.
\end{abstract}

\section{INTRODUCTION}

The modular class of a Poisson manifold is an obstruction lying in the first Poisson cohomology to the existence of a volume form invariant with respect to Hamiltonian flows, [Ko, We3]. Hence, the modular class should be closely related to the "holonomy" of the Poisson manifold. The reason is that the trace of the linearized holonomy operators can be viewed as a first obstruction to the existence of a transversal holonomy-invariant volume form. The connection between the holonomy and the modular class can be easily made rigorous and explicit when the Poisson manifold is regular and the notion of holonomy is known from the theory of foliations (see, e. g., [Godb]).

In the present paper we introduce the linear holonomy $h$ on Poisson manifolds $P$ without making any regularity assumptions on the Poisson structure $\pi$ of $P$ (Section 2.1). In the regular case, $h$ is equivalent to the linearized holonomy of the symplectic foliation of $\pi$. However, the objects along which the Poisson holonomy $h$ is defined are not ordinary loops tangent to the leaves, but rather "cotangent loops". These are the mappings $\alpha: S^{1} \rightarrow T^{*} P$ such that $\pi^{\#} \alpha$ is the projection of the derivative $\alpha^{\prime}$ to $T P$. In contrast with the holonomy of regular foliations, the Poisson linear holonomy for singular

Date: December 27, 1998.

The work was partially supported by the NSF and by the faculty research funds of the UC Santa Cruz. 
Poisson structures is not homotopy invariant in the standard sense, but its certain counterpart is (Section 2.2).

We show that the determinant of the linear Poisson holonomy is determined by the integral of the modular class over the cotangent loop. In fact, the determinant is equal to the exponential function of the integral; see Section 3.2, where we also recall the definition of the modular class. This result requires defining the integral of a vector field over a cotangent loop, which is done in Section 3.1.

In the second part of the paper we focus on the question of Morita invariance of the modular class. More specifically, it is known that the first Poisson cohomology space is an invariant of Morita equivalence; see [GL. This naturally leads to the problem whether the modular class is an invariant of Morita equivalence or not. We show (Theorem 4.2) that the modular class is such an invariant for locally unimodular Poisson manifolds, i. e., for manifolds which locally admit a volume form conserved by Hamiltonian flows. In particular, the modular class is Morita invariant for regular Poisson manifolds. Furthermore, a Poisson manifold which is Morita equivalent to a unimodular manifold is also unimodular.

The definition and basic properties of Morita equivalence are recalled in Section 4.1. Theorem 4.2 is proved in Section 5. Basic definitions and results from Poisson geometry used in this paper can be found in $\mathrm{CW}, \mathrm{Va}$.

Acknowledgments. We would like to thank Richard Montgomery for calling our attention to the proof of Liouville's theorem in [SM] and Alan Weinstein for numerous useful remarks.

\section{Linear Poisson Holonomy}

2.1. Linear Poisson holonomy. Consider a Poisson manifold $(P, \pi)$. A cotangent loop in $P$ is a smooth mapping $\alpha: S^{1} \rightarrow T^{*} P$ such that

$$
\pi^{\#}(\alpha)=(\operatorname{pr}(\alpha))^{\prime} .
$$

Here, pr: $T^{*} P \rightarrow P$ is the natural projection, $\pi^{\#}: T^{*} P \rightarrow T P$ is the pairing with $\pi$, and the prime, as usual, denotes the derivative with respect to the time.

The projection $\gamma=\operatorname{pr}(\alpha)$ of the cotangent loop $\alpha$ is necessarily tangent to a leaf of the symplectic foliation of $P$. Moreover, as is easy to see, every tangent loop $\gamma$ has a cotangent lift $\alpha$, i. e., a cotangent loop $\alpha$ such that $\pi^{\#}(\alpha)=\gamma^{\prime}$. The lift $\alpha$ is never unique, unless $P$ is symplectic near $\gamma$. In what follows, we will always denote $\operatorname{pr}(\alpha)$ by $\gamma$.

Let us define the linear holonomy along a cotangent loop $\alpha$. Pick a family of closed one-forms $\tilde{\alpha}_{t}$ such that $\tilde{\alpha}_{t}(\gamma(t))=\alpha(t)$. Then $\gamma$ is an integral curve of the time-dependent vector field $v_{t}=\pi^{\#} \tilde{\alpha}_{t}$. Parameterize $S^{1}$ as $\mathbb{R} / \mathbb{Z}$. Let $\phi_{t}$ be the time-dependent flow of $v_{t}$. Then the linearization of the time-one flow $d \phi_{1}: T_{\gamma(0)} P \rightarrow T_{\gamma(0)} P$ preserves the tangent space $T_{\gamma(0)} F_{\gamma(0)}$ to the leaf $F_{\gamma(0)}$ through $\gamma(0)$ of the symplectic foliation. 
Denote by $N_{\gamma}(0)$ the normal space to $F_{\gamma(0)}$ at $\gamma(0)$, i. e.,

$$
N_{\gamma(0)}=T_{\gamma(0)} P / T_{\gamma(0)} F_{\gamma(0)} \text {. }
$$

The linearization $d \phi_{1}$ induces a linear map $h(\alpha): N_{\gamma(0)} \rightarrow N_{\gamma(0)}$. Recall that $N_{\gamma(0)}$ carries a canonical linear Poisson structure which is the linearization of the normal Poisson structure at $\gamma(0)$; see We1].

Proposition 2.1. The linear map $h(\alpha)$ is Poisson and independent of the choice of $\tilde{\alpha}$, for a fixed $\alpha$.

Definition 2.2. The map $h(\alpha)$ is called the linear Poisson holonomy along the cotangent loop $\alpha$.

Example 2.3. Assume that $\pi$ is regular, i. e., of constant rank, near $\gamma$. Then the symplectic foliation of $\pi$ is regular near $\gamma$ and $h(\alpha)$ is just the linearization of the holonomy along $\gamma$ in the sense of the theory of foliations. (See, e.g., Godb.) We see that in this case $h(\alpha)$ is determined entirely by $\gamma$ and the symplectic foliation, rather than by $\alpha$ and $\pi$, and that $h(\alpha)$ is an invariant of the homotopy of $\gamma$ in the leaf.

Example 2.4. Let $P=\mathfrak{g}^{*}$, where $\mathfrak{g}$ is a finite-dimensional Lie algebra, and let $\alpha: S^{1} \rightarrow T_{0}^{*} \mathfrak{g}^{*}=\mathfrak{g}$ be a constant mapping. Then $N_{\gamma(0)}=\mathfrak{g}^{*}$ and, as one can easily check, $h(\alpha)=\exp \operatorname{ad}_{\alpha}^{*}=\operatorname{Ad}_{\exp (\alpha)}^{*}$. Hence, in this case, $h(\alpha)$ depends on $\alpha$ and $\pi$ rather than just on $\gamma$ and the symplectic foliation. Moreover, in contrast with Example 2.3, $h(\alpha)$ is not homotopy invariant in the conventional sense, i. e., for deformations of $\alpha$ in the class of cotangent loops over a fixed symplectic leaf. In fact, $h(\alpha)$ may change even when $\alpha$ is deformed as a constant cotangent loop over $\gamma=0$.

Remark 2.5. For a singular foliation, Dazord, [Da1], introduces the holonomy along a tangent loop as a mapping of the space of leaves of the induced foliation on the normal slice to the leaf. The linear version of such holonomy is defined using the linearized normal foliation, [Da2]. Linear Poisson holonomy and reduced linear Poisson holonomy, defined in Section 2.2, seem to be essentially unrelated, except some obvious cases, to holonomy for singular foliations.

Proof of Proposition 2.1. Let $\tilde{\alpha}_{t}$ and $\tilde{\alpha}_{t}+\beta_{t}$ be two closed time-dependent one-forms used as extensions of $\alpha$ so that

$$
\pi^{\#} \beta_{t}(\gamma(t))=0 .
$$

Step 1. Assume first that $\beta_{t}$ is localized in space. In other words, suppose that there exists a point $t_{0} \in S^{1}$ and a neighborhood $U$ of $\gamma\left(t_{0}\right)$ such that $\beta_{t}$ is supported in $U$ for all $t$. Assume that $U$ is small enough so that the splitting theorem, We1, applies. Thus, $U=F \times N$ and $\pi=\pi_{F}+\pi_{N}$, where $F$ is a neighborhood of $\gamma\left(t_{0}\right)$ in the symplectic leaf and $N$ is a normal slice. 
Here $\pi_{F}$ and $\pi_{N}$ denote, respectively, the tangent and normal components of $\pi$. Recall that $\pi_{N}$ vanishes along the leaf $F$.

Then, in $U$,

$$
\pi^{\#}\left(\tilde{\alpha}_{t}+\beta_{t}\right)-\pi^{\#} \tilde{\alpha}_{t}=\pi_{F}^{\#} \beta_{t}+\pi_{N}^{\#} \beta_{t} .
$$

The first term on the right hand side is tangent to the $F$-component. The second term vanishes at $\gamma(t)$ together with its linearization because both $\pi_{N}$ and $\beta_{t}$ vanish at $\gamma(t)$. Hence none of the terms on the right hand side contributes to the normal component of the linearized flow. As a result, the linear holonomy defined by means of $\tilde{\alpha}_{t}$ is the same as that defined via $\tilde{\alpha}_{t}+\beta_{t}$.

Step 2. Let us reduce the general case to that where $\beta_{t}$ is localized. Let $\beta_{t}$ be arbitrary.

First, observe that only the restriction of $\tilde{\alpha}_{t}+\beta_{t}$ to a neighborhood of $\gamma(t)$ effects the flow $\phi_{t}$. Thus we can assume that for every $t \in S^{1}$ the form $\beta_{t}$ is supported in a small neighborhood $U_{t}$ of $\gamma(t)$.

Furthermore, by compactness, there exists an open cover of $S^{1}$ by intervals $I_{l}, l=1, \ldots, k$, such that for every $l$ and every $t \in I_{l}$ the form $\beta_{t}$ is supported in an open set $U^{(l)}$ to which the splitting theorem applies. Let $f_{l}$ be a smooth partition of unity on $S^{1}$ subordinated to the cover $\left\{I_{l}\right\}$. Set

$$
\tilde{\alpha}_{t}^{(l)}=\tilde{\alpha}_{t}+\left(f_{1}+\ldots+f_{l}\right) \beta_{t}
$$

with $\tilde{\alpha}_{t}^{(0)}=\tilde{\alpha}_{t}$. Then $\tilde{\alpha}_{t}^{(l-1)}$ and $\tilde{\alpha}_{t}^{(l)}$ for $l=1, \ldots, k$ differ by the form $f_{l} \beta_{t}$ which satisfies (2) and is localized in $U^{(l)}$. The argument of the first step applies to these forms and therefore $\tilde{\alpha}_{t}^{(l-1)}$ and $\tilde{\alpha}_{t}^{(l)}$ give rise to the same linear holonomy. We conclude by induction that this is also true for $\tilde{\alpha}_{t}^{(0)}=\tilde{\alpha}_{t}$ and $\tilde{\alpha}_{t}^{(k)}=\tilde{\alpha}_{t}+\beta_{t}$.

The fact that $h(\alpha)$ is Poisson is an immediate consequence of that $\phi_{t}$ preserves the Poisson structure which, in turn, follows from that $\tilde{\alpha}_{t}$ is closed.

Remark 2.6. When $\gamma$ is an embedding, the form $\tilde{\alpha}_{t}$ can be chosen independent of time. However, if $\gamma$ is not one-to-one (e. g., as in Example 2.4), such a choice may not be possible. Also note that a choice of non-closed forms $\tilde{\alpha}_{t}$ would still lead to the same linear Poisson mapping $h(\alpha)$ as above. However, for what follows, it is more convenient to assume that $\tilde{\alpha}_{t}$ are closed.

Remark 2.7. Independence of Parameterization. The linear holonomy $h(\alpha)$ is independent of parameterization in the following sense. For an orientation preserving diffeomorphism $\varphi: S^{1} \rightarrow S^{1}$, set $\alpha^{\varphi}=\varphi^{\prime} \cdot \alpha \circ \varphi$. Then $\alpha^{\varphi}$ also satisfies (11), and hence $\alpha^{\varphi}$ is a cotangent loop. It is not hard to see that $h(\alpha)=h\left(\alpha^{\varphi}\right)$, where for the sake of simplicity we have assumed that $\varphi(0)=0$. 
Remark 2.8. Let $\alpha:[a, b] \rightarrow T^{*} P$ be a smooth cotangent path, i. e., a curve satisfying (11), but not necessarily closed. Then similarly to the above, one can define the linear Poisson holonomy $h(\alpha): N_{\gamma(a)} \rightarrow N_{\gamma(b)}$, where as before $\gamma$ is the projection of $\alpha$ to $P$. The holonomy $h(\alpha)$ is again independent of parameterization in the sense of Remark 2.7. Note that our definition of cotangent paths is a particular case of the definition of admissible curves introduced for arbitrary algebroids in We2].

It is easy to extend the linear holonomy to piecewise smooth paths. Then $h$ becomes multiplicative with respect to "composition" of paths. More explicitly, let $\alpha_{1}:[a, b] \rightarrow T^{*} P$ and $\alpha_{2}:[b, c] \rightarrow T^{*} P$ be two piecewise smooth cotangent paths such that $\gamma_{1}(b)=\gamma_{2}(b)$. We have a naturally defined cotangent path $\alpha_{1} \alpha_{2}:[a, c] \rightarrow T^{*} P$ and

$$
h\left(\alpha_{1} \alpha_{2}\right)=h\left(\alpha_{2}\right) h\left(\alpha_{1}\right) .
$$

Remark 2.9. The main motivation for the definitions of this section is the general principle that on a Poisson manifold the roles of the tangent and cotangent bundles are often switched. In other words, on a Poisson manifold covectors should, in some cases, be given the role that vectors play on a smooth manifold. For example, a cotangent path is just a Poisson analogue of an ordinary curve in a smooth manifold. In Section 3 we will see another application of this principle: the definition of the integral of a vector field along a cotangent path.

Remark 2.10. The Bott connection. The infinitesimal counterpart of Poisson holonomy is the following analogue of the Bott connection. Let $\beta$ be a one-form along a leaf $F$ of the symplectic foliation. Assume that $\left.\beta\right|_{F}=0$, i. e., $\beta$ is a section of the normal bundle of $F$. For $p \in F$ and $\alpha \in T_{p}^{*} P$, we set $\nabla_{\alpha} \beta=\left.L_{\pi \# \tilde{\alpha}} \tilde{\beta}\right|_{T_{p} P}$, where $\tilde{\alpha}$ is an extension of $\alpha$ to a neighborhood of $p$ and $\tilde{\beta}$ is a local extension of $\beta$. It is easy to see that $\nabla_{\alpha} \beta$ is well defined, "normal" to $F$, and that $\nabla$ has the properties of the ordinary Bott connection. This definition is a particular case of the construction of the Bott connection for algebroids given in [ELW, Appendix A].

2.2. Homotopy non-invariance of the holonomy. As we have already seen in Example 2.4, linear holonomy is not homotopy invariant when the Poisson structure is singular. In this section, we will show how to turn linear holonomy into a homotopy invariant by reducing the information carried by the holonomy operators.

Let $\mathfrak{g}$ be a finite-dimensional Lie algebra. Denote by Aut $\left(\mathfrak{g}^{*}\right)$ the group of linear isomorphisms $\mathfrak{g}^{*} \rightarrow \mathfrak{g}^{*}$ which are dual to Lie algebra automorphisms $\mathfrak{g} \rightarrow \mathfrak{g}$. Equivalently, Aut $\left(\mathfrak{g}^{*}\right)$ is the group of linear Poisson isomorphisms $\mathfrak{g}^{*} \rightarrow \mathfrak{g}^{*}$. The group $\operatorname{Aut}\left(\mathfrak{g}^{*}\right)$ contains the normal subgroup $\operatorname{Inn}\left(\mathfrak{g}^{*}\right)$ of inner automorphisms. The group $\operatorname{Inn}\left(\mathfrak{g}^{*}\right)$ is comprised of the automorphisms of the form $\exp \operatorname{ad}_{\alpha}^{*}$, where $\alpha \in \mathfrak{g}$. Alternatively, we can define $\operatorname{Inn}\left(\mathfrak{g}^{*}\right)$ as the group of linear Hamiltonian isomorphisms. The quotient $\operatorname{Out}\left(\mathfrak{g}^{*}\right)=$ 
$\operatorname{Aut}\left(\mathfrak{g}^{*}\right) / \operatorname{Inn}\left(\mathfrak{g}^{*}\right)$ is the group of outer automorphisms of $\mathfrak{g}^{*}$. Note that the Lie algebra of Out $\left(\mathfrak{g}^{*}\right)$ is $H^{1}(\mathfrak{g} ; \mathfrak{g})$, which can also be thought of as the first "linear Poisson cohomology" of $\mathfrak{g}^{*}$. For an element $h \in \operatorname{Aut}\left(\mathfrak{g}^{*}\right)$, denote the class of $h$ in $\operatorname{Out}\left(\mathfrak{g}^{*}\right)$ by $\bar{h}$.

Recall that for a normal space $N_{x}$ at $x \in P$ to the symplectic leaf through $x$, the dual space $N_{x}^{*}$ is a Lie algebra. Applying the above construction to $\mathfrak{g}^{*}=N_{x}$, so that $\mathfrak{g}=N_{x}^{*}$, we obtain the group Out $\left(N_{x}\right)$. For a cotangent loop $\alpha$, denote by $\bar{h}(\alpha)$ the equivalence class of the linear holonomy of $h(\alpha)$ in $\operatorname{Out}\left(N_{\gamma(0)}\right)$. We call $\bar{h}(\alpha)$ the reduced linear holonomy.

Example 2.11. If $\pi$ is regular, $N_{x}^{*}$ is commutative. Hence, in this case,

$$
\operatorname{Out}\left(N_{x}\right)=\operatorname{Aut}\left(N_{x}\right)=\operatorname{GL}\left(N_{x}\right) \text {. }
$$

On the other hand, if $N_{x}^{*}$ is semisimple, $\operatorname{Inn}\left(N_{x}\right)$ is the identity connected component in $\operatorname{Aut}\left(N_{x}\right)$, and so $\operatorname{Out}\left(N_{x}\right)$ is discrete.

Let $\alpha^{s}: S^{1} \rightarrow P$ be a family of cotangent loops parameterized by $s \in$ $(-1,1)$. For the sake of simplicity we assume that the initial point $x=\gamma^{s}(0)$, where $\gamma^{s}=\operatorname{pr}\left(\alpha^{s}\right)$, is fixed, i. e., independent of $s$. Note that, in particular, this implies that all $\gamma^{s}$ lie in the same symplectic leaf.

Theorem 2.12 (Homotopy invariance). The reduced linear holonomy is homotopy invariant: $\bar{h}\left(\alpha^{s}\right) \in \operatorname{Out}\left(N_{x}\right)$ is independent of $s$.

We will prove a result more general than Theorem 2.12. Let $x$ and $y$ be two points on the same leaf of $P$. Denote by $E_{x, y}$ the linear space of linear Poisson operators $N_{x} \rightarrow N_{y}$. The group $\operatorname{Inn}\left(N_{x}\right)$ acts on $E_{x, y}$ from the right and $\operatorname{Inn}\left(N_{y}\right)$ acts from the left. The orbit spaces of both actions coincide: every $\operatorname{Inn}\left(N_{x}\right)$-orbit on $E_{x, y}$ is also an $\operatorname{Inn}\left(N_{y}\right)$-orbit and vice versa. Denote the resulting orbit space by $\bar{E}_{x, y}$, i. e.,

$$
\bar{E}_{x, y}=E_{x, y} / \operatorname{Inn}\left(N_{x}\right)=\operatorname{Inn}\left(N_{y}\right) \backslash E_{x, y} .
$$

By definition, for a cotangent path $\alpha:[a, b] \rightarrow T^{*} P$ with end-points $x=\gamma(a)$ and $y=\gamma(b)$, the reduced holonomy $\bar{h}(\alpha) \in \bar{E}_{x, y}$ is the equivalence class of the holonomy $h(\alpha)$.

Consider a homotopy $\alpha^{s}:[a, b] \rightarrow T^{*} P$, where $s \in(-1,1)$, with fixed end-points $x$ and $y$, i. e., such that $\gamma^{s}(a)=x$ and $\gamma^{s}(b)=y$ for all $s$. Note that the paths $\gamma^{s}$ are necessarily contained in the same leaf. Theorem 2.12 is an immediate consequence of the following

Proposition 2.13. The reduced holonomy $\bar{h}\left(\alpha^{s}\right) \in \bar{E}_{x, y}$ is independent of $s \in(-1,1)$.

It is easy to see that cotangent lifts of a fixed tangent curve, closed or not, are homotopic to each other. Thus, we have

Corollary 2.14. The reduced holonomy $\bar{h}(\alpha)$ is determined completely by the projection $\operatorname{pr}(\alpha)$. 
Proof of Proposition 2.13. Step 1. Assume first that, in the notations of the proof of Proposition 2.1, all $\gamma^{s}([a, b])$ are contained in a small open set $U=F \times N$ to which the splitting theorem applies and such that $F$ is an open ball.

Then $\alpha_{s}$ can be decomposed as $\left(\pi_{F}^{\#}\right)^{-1}\left(\gamma^{s}\right)^{\prime}+\nu^{s}$, where the first term is the tangent component and the second term is the normal component, i. e., $\pi^{\#} \nu^{s}=0$. It is clear that the tangent component of the variation $\alpha^{s}$ has no effect on the holonomy. Hence, we may assume that $F$ is a point, $\gamma$ is a constant path at the singular point $x=y \in N$, and $\nu^{s}(t)=\alpha^{s}(t) \in T_{x}^{*} N$. Then, similarly to Example 2.4,

$$
h\left(\alpha^{s}\right)=\exp \left(\int_{a}^{b} \operatorname{ad}_{\alpha^{s}(t)}^{*} d t\right) .
$$

In particular, $h\left(\alpha^{s}\right) \in \operatorname{Inn}\left(N_{x}\right)$ and hence $\bar{h}\left(\alpha^{s}\right)$ is independent of $s$.

Thus, we have proved that the reduced holonomy $\bar{h}(\alpha)$ is independent of $\alpha$ connecting $x$ and $y$ in $U$.

Step 2. Clearly, it suffices to prove that $\bar{h}\left(\alpha^{0}\right)=\bar{h}\left(\alpha^{\epsilon}\right)$ for an arbitrarily small $\epsilon>0$. Pick $\epsilon>0$ and a partition

$$
a=t_{0}<t_{1}<\cdots<t_{k}<t_{k+1}=b
$$

of $[a, b]$ so that for all $j=0, \ldots, k$, the homotopy $\gamma^{s}\left(\left[t_{j}, t_{j+1}\right]\right), s \in[0, \epsilon]$, is contained in a neighborhood $U_{j}$ to which Step 1 applies.

Let $\alpha_{j}$ be the restriction of $\alpha^{0}$ to $\left[t_{j}, t_{j+1}\right]$. The path $\alpha_{j}$ is homotopic with fixed end-points to the path which is the restriction of $\alpha^{s}(t)$ to the other three edges of the rectangle $\left[t_{j}, t_{j+1}\right] \times[0, \epsilon]$. However, this path is not cotangent, because $s \mapsto \alpha^{s}(t)$ is not, in general, cotangent for a fixed $t$. Hence, we need to slightly modify its definition. Let $\beta_{j}:\left[t_{j}-1, t_{j+1}+1\right] \rightarrow$ $T^{*} U_{j}$ be a piecewise smooth cotangent path defined as the composition of the following three:

- On the interval $\left[t_{j}-1, t_{j}\right]$, the tangent component of $\beta_{j}$ is determined by $s \mapsto \gamma^{s}\left(t_{j}\right)$. The normal component is the linear interpolation between the normal components of $\alpha^{0}\left(t_{j}\right)$ and $\alpha^{\epsilon}\left(t_{j}\right)$. In other words,

$$
\beta_{j}(\tau)=\left(\pi_{F}^{\#}\right)^{-1}\left(\frac{d \gamma^{s}\left(t_{j}\right)}{d s}\right)+\left(\frac{s}{\epsilon}+1\right) \nu^{0}\left(t_{j}\right)+\frac{s}{\epsilon} \nu^{\epsilon}\left(t_{j}\right),
$$

where $s=\epsilon\left(\tau-t_{j}+1\right)$ with $\tau \in\left[t_{j}-1, t_{j}\right]$ and, as before, $\nu^{s}(t)$ denotes the normal component of $\alpha^{s}(t)$.

- For $\tau \in\left[t_{j}, t_{j+1}\right]$, the path $\beta_{j}(\tau)$ is just $\alpha^{\epsilon}(\tau)$.

- On $\left[t_{j+1}, t_{j+1}+1\right]$, the path $\beta_{j}$ is defined in the same manner as on the interval $\left[t_{j}-1, t_{j}\right]$, but with the path $s \mapsto \gamma^{s}\left(t_{j+1}\right)$ traversed backward:

$$
\beta_{j}(\tau)=\left(\pi_{F}^{\#}\right)^{-1}\left(\frac{d \gamma^{s}\left(t_{j+1}\right)}{d s}\right)+\left(\frac{s}{\epsilon}+1\right) \nu^{\epsilon}\left(t_{j+1}\right)+\frac{s}{\epsilon} \nu^{0}\left(t_{j+1}\right)
$$

where $\tau \in\left[t_{j+1}, t_{j+1}+1\right]$ and $s=\epsilon\left(t_{j+1}-\tau\right)$. 
The path $\beta_{j}$ has the same end-points as $\alpha_{j}$ and both paths are contained in $U_{j}$. By Step $1, \bar{h}\left(\beta_{j}\right)=\bar{h}\left(\alpha_{j}\right)$.

It is clear that $\alpha^{0}$ is the composition $\alpha_{0} \alpha_{1} \cdots \alpha_{k}$. Furthermore, note that $h\left(\beta_{0} \beta_{1} \ldots \beta_{k}\right)=h\left(\beta^{\epsilon}\right)$, because for each $j$ the holonomy along the third part of $\beta_{j}$ is canceled by the holonomy along the first part of $\beta_{j+1}$. (Strictly speaking, to take this composition we need to reparameterize each $\beta_{j}$ so as to turn its domain into $\left[t_{j}, t_{j+1}\right]$.) As a consequence, $\bar{h}\left(\alpha^{\epsilon}\right)=\bar{h}\left(\alpha^{0}\right)$.

Remark 2.15. The material of this section and most of Section 3 extend essentially word-for-word to arbitrary algebroids when the definitions and results from ELW are taken into account. The linear holonomy defined here appears to be related to the adjoint "representation" of a groupoid, [ELW, Appendix B].

\section{Linear Holonomy and the Modular Class}

3.1. Linear integrals on Poisson manifolds. Let $\alpha:[a, b] \rightarrow T^{*} P$ be a smooth cotangent path and $v$ a vector field on $P$. Define

$$
\int_{\alpha} v=-\int_{a}^{b} \alpha_{\gamma(t)}(v(\gamma(t)) d t
$$

where as before $\gamma$ is the projection of $\alpha$ to $P$, i. e., $\gamma=\operatorname{pr}(\alpha)$. The following proposition summarizes the properties of the integral which are important for what follows.

Proposition 3.1. 1. Assume that $v=\pi^{\#} \beta$. Then

$$
\int_{\alpha} v=\int_{\gamma} \beta
$$

2. Let $v$ be a Hamiltonian vector field with Hamiltonian $f$, $i$. e., $v=\pi^{\#} d f$. Then

$$
\int_{\alpha} v=f(\gamma(b))-f(\gamma(a))
$$

Proof. The second assertion follows immediately from the first one. To prove the first assertion, note that $\alpha\left(\pi^{\#} \beta\right)=-\beta\left(\pi^{\#} \alpha\right)$. Thus

$$
\int_{\alpha} \pi^{\#} \beta=-\int_{a}^{b} \alpha\left(\pi^{\#} \beta(t)\right) d t=\int_{a}^{b} \beta\left(\pi^{\#} \alpha(t)\right) d t=\int_{a}^{b} \beta\left(\gamma^{\prime}(t)\right) d t=\int_{\gamma} \beta .
$$

Recall that a vector field $v$ is said to be Poisson if $L_{v} \pi=0$. For example, Hamiltonian vector fields are Poisson. The first Poisson cohomology of $P$ is the quotient of the space of Poisson vector fields on $P$ by the space of Hamiltonian vector fields:

$$
H_{\pi}^{1}(P)=\frac{\text { Poisson }}{\text { Hamiltonian }}
$$


Corollary 3.2. Assume that $\alpha$ is a cotangent loop. Then the integral along $\alpha$ gives rise to a linear mapping

$$
\int_{\alpha}: H_{\pi}^{1}(P) \rightarrow \mathbb{R}
$$

Remark 3.3. The integral along cotangent paths can be extended to piecewise smooth paths in the standard way.

Example 3.4. Similarly to the holonomy (Example 2.4), the integral of a Poisson vector field over a cotangent loop is not a homotopy invariant when the homotopy is understood as a deformation of the cotangent loop in the class of cotangent loops (as in Section 2.2). To be more precise, consider a fixed Poisson vector field $v$ and a family of cotangent loops $\alpha_{\tau}, \tau \in[0,1]$. Then $\int_{\alpha_{\tau}} v$ does not have to be independent of $\tau$. For example, let $\pi=0$ and let $\alpha=$ const, i. e., $\alpha(t)$ is independent of $t \in S^{1}$. (Constants are the only cotangent loops for the zero Poisson structure.) It is easy to see that the integral is equal to $\alpha(v)$ which clearly is not a homotopy invariant in the above sense, even over a fixed symplectic leaf.

This example shows that the above naive definition of homotopy is not a "correct" extension of this notion to the Poisson category.

Note also that the integral becomes homotopy invariant when $v=\pi^{\#} \beta$ for a closed one-form $\beta$. This follows from Proposition 3.1.

3.2. The holonomy and the modular class. The modular class of a Poisson manifold $P$ is the obstruction to the existence of a volume form on $P$ which is invariant with respect to Hamiltonian flows. More explicitly, let $\mu$ be a volume form on $P$. As shown in [Ko, We3], there exists a unique vector field $v_{\mu}$, called the modular vector field, such that for every smooth function $f$ on $P$, we have

$$
\operatorname{div}_{\mu} X_{f}=L_{v_{\mu}} f
$$

where $X_{f}$ is the Hamiltonian vector field of $f$, i. e., $X_{f}=\pi^{\#} d f$, and $\operatorname{div}_{\mu}$ is the divergence taken with respect to $\mu$, i. e., $\operatorname{div}_{\mu} X_{f}=L_{X_{f}} \mu$. Alternatively, $v_{\mu}$ can be characterized by the condition that

$$
\operatorname{div}_{\mu} \pi^{\#} \beta=\iota_{v_{\mu}} \beta
$$

for every closed one-form $\beta$ on $P$. The vector field $v_{\mu}$ is Poisson. Furthermore,

$$
v_{g \mu}=v_{\mu}-X_{\ln g}
$$

for any positive smooth function $g$ on $P$. Thus the first Poisson cohomology class of $v_{\mu}$ is independent of $\mu$. This class, denoted henceforth by $\bmod (P)$, is called the modular class of $P$.

The existence of $v_{\mu}$ was first pointed out by Koszul in [Ko]. The modular class was introduced by A. Weinstein, $\overline{\mathrm{We} 3}$. Both the modular class and the modular vector field are thoroughly studied in [We3] as a part of the 
program of the analysis of connections between Poisson manifolds and operator algebras. From the perspective of quantization, the raison d'être for the Poisson modular class is that the flow of the modular vector field is the semi-classical limit of the modular automorphism group for von Neumann algebras. (See [C, We3] for more details.)

When the manifold $P$ is symplectic, the Liouville form is preserved by Hamiltonian flows, and hence the modular class of $P$ is zero. Similarly, on a general Poisson manifold $P$, since Hamiltonian flows preserve the leaf-wise Liouville form $\bmod (P)$ is an obstruction to the existence of an invariant "normal" volume form. This indicates that there is a strong connection between $\bmod (P)$ and holonomy. (See, also, We3, ELW.) For example, it is easy to prove that, when $P$ is regular, $\bmod (P)=0$ implies that there is a normal (i. e. transversal) volume form which is holonomy-invariant. In particular, the linearized holonomy has unit determinant. Our goal now is to establish a connection between $\operatorname{det} h(\alpha)$ and $\bmod (P)$ for all Poisson manifolds.

Theorem 3.5. Let $\alpha$ be a cotangent loop in a Poisson manifold P. Then

$$
\operatorname{det} h(\alpha)=\exp \left(\int_{\alpha} \bmod (P)\right)
$$

Note that the right hand side of $(6)$ is just $\int_{\alpha} v_{\mu}$, since $\bmod (P)$ is the cohomology class of the modular vector field $v_{\mu}$.

Recall that $P$ is said to be unimodular if $\bmod (P)=0$, We3. This terminology is motivated by the fact, We3, that $\bmod \left(\mathfrak{g}^{*}\right)=0$ if and only if $\mathfrak{g}$ is unimodular.

Corollary 3.6. Assume that $P$ is unimodular. Then $\operatorname{det} h(\alpha)=1$ for any cotangent loop $\alpha$.

Proof of Theorem 3.5. Let us first state (6) in a more general form. Fix a volume form $\mu$ on $P$. Let $\alpha:[a, b] \rightarrow P$ be a cotangent path (not necessarily closed). Then according to Remark 2.8 the holonomy along $\alpha$ is a linear map $h(\alpha): N_{\gamma(a)} \rightarrow N_{\gamma(b)}$. The volume form $\mu$ together with the leaf-wise Liouville volume form give rise to linear volume forms on $N_{\gamma(a)}$ and $N_{\gamma(b)}$.

Proposition 3.7. For any cotangent path $\alpha$, we have

$$
\operatorname{det} h(\alpha)=\exp \left(\int_{\alpha} v_{\mu}\right),
$$

where the determinant is taken with respect to the linear volume forms induced by $\mu$ on $N_{\gamma(a)}$ and $N_{\gamma(b)}$.

Theorem 3.5 is a consequence of Proposition 3.7, for the determinant is independent of the choice of $\mu$, when $\alpha$ is closed.

Proof of Proposition 3.X. The proposition follows from a version of the classical Liouville theorem. Let us recall the theorem. Let $P$ be a manifold 
with a volume form $\mu$ and let $\gamma:[a, b] \rightarrow P$ be an integral curve of a timedependent vector field $w_{t}$ on $P$. Denote by $\phi_{t}$ the local time-dependent flow of $w_{t}$ on $P$. The linearization of $\phi_{t}$ along $\gamma$ gives rise to a linear mapping $\Phi: T_{\gamma(a)} P \rightarrow T_{\gamma(b)} P$. According to Liouville's theorem,

$$
\operatorname{det} \Phi=\exp \left(\int_{a}^{b}\left(\operatorname{div}_{\mu} w_{t}\right)(\gamma(t)) d t\right)
$$

where the determinant is taken with respect to $\mu$. (See, e. g., SM, p. 142] for a proof.)

To derive (7) from (8), consider a time-dependent closed one-form $\tilde{\alpha}_{t}$ which extends $\alpha$. Then $\gamma=\operatorname{pr}(\alpha)$ is an integral curve of $w_{t}=\pi^{\#} \tilde{\alpha}_{t}$.

First, let us show that the left hand side of $(7)$ is equal to that of (8). The linearization $\Phi$ preserves the splitting of $T P$ into the components tangent and normal to the leaves. The tangent component of $\Phi$ has determinant one because the standard symplectic volume form on the leaves is preserved by the flow. (This is another version of Liouville's theorem.) The normal part is equal to $h(\alpha)$, and hence $\operatorname{det} h(\alpha)=\operatorname{det} \Phi$.

To equate the right hand sides of both equations, it suffices to observe that, by (5),

$$
\int_{\alpha} v_{\mu}=\int_{a}^{b} \tilde{\alpha}_{t}\left(v_{\mu}(\gamma(t))\right) d t=\int_{a}^{b} \operatorname{div}_{\mu}\left(\pi^{\#} \tilde{\alpha}_{t}\right)(\gamma(t)) d t
$$

Since by definition $w_{t}=\pi^{\#} \tilde{\alpha}_{t}$, we see that

$$
\int_{\alpha} v_{\mu}=\int_{a}^{b}\left(\operatorname{div}_{\mu} w_{t}\right)(\gamma(t)) d t
$$

This concludes the proof of Proposition 3.7 and thus of Theorem 3.5.

Remark 3.8. It is worth pointing out that linear holonomy carries less information than ordinary holonomy would. To illustrate this point, let us focus on the case of a regular Poisson structure. Then the ordinary holonomy $H$ is defined and $h(\alpha)$ is just the linearization of the holonomy $H(\gamma)$ along $\gamma$ (Example 2.3), and so $h$ is determined by $H$ but not vice versa.

For example, consider the standard Reeb foliation on $S^{3}$ (see, e.g., Godb, Section I.3.14]) with $\pi$ given by the leafwise area form. It is easy to see that $h(\alpha)=$ id for any loop $\alpha$ and hence $\int_{\alpha} \bmod (P)=0$. On the other hand, the genuine holonomy $H(\gamma)$ is non-trivial for a vanishing cycle $\gamma$. Moreover, $\bmod (P) \neq 0$. For, otherwise, the Reeb foliation would admit a transversal holonomy-invariant volume form. It is clear that such a form does not exist. (See also We3.)

Note also that $\bmod (P)$ for the Reeb foliation gives an example of a nonzero (tangential) class in $H_{\pi}^{1}(P)$ whose integral over any cotangent loop is zero. In particular, the restriction of this class to every leaf is zero. 


\section{Morita Equivalence And the Modular Class}

4.1. Morita equivalence. Following We1, recall that a full dual pair $P_{1} \stackrel{\rho_{1}}{\leftarrow} W \stackrel{\rho_{2}}{\rightarrow} P_{2}$ consists of two Poisson manifolds $\left(P_{1}, \pi_{1}\right)$ and $\left(P_{2}, \pi_{2}\right)$, a symplectic manifold $W$, and two submersions $\rho_{1}: W \rightarrow P_{1}$ and $\rho_{2}: W \rightarrow P_{2}$ such that $\rho_{1}$ is Poisson, $\rho_{2}$ is anti-Poisson, and the fibers of $\rho_{1}$ and $\rho_{2}$ are symplectic orthogonal to each other. A Poisson (or anti-Poisson) mapping is said to be complete if the pull-back of a complete Hamiltonian flow under this mapping is complete. A full dual pair is called complete if both $\rho_{1}$ and $\rho_{2}$ are complete. The Poisson manifolds $P_{1}$ and $P_{2}$ are Morita equivalent if there exists a complete full dual pair $P_{1} \stackrel{\rho_{1}}{\leftarrow} W \stackrel{\rho_{2}}{\rightarrow} P_{2}$ such that $\rho_{1}$ and $\rho_{2}$ both have connected and simply connected fibers. The notion of Morita equivalence of Poisson manifolds was introduced and studied by $\mathrm{P} . \mathrm{Xu}$, $\mathrm{Xu}$, as a classical analogue of the Morita equivalence of $C^{*}$-algebras (see, e. g., [Co ).

Let us summarize some properties of Poisson manifolds $P_{1}$ and $P_{2}$ forming a complete full dual pair $P_{1} \stackrel{\rho_{1}}{\leftarrow} W \stackrel{\rho_{2}}{\rightarrow} P_{2}$ with connected fibers.

1. [We1]. For a symplectic leaf $F \subset P_{1}$, the projection $\rho_{2}\left(\rho_{1}^{-1}(F)\right)$ is a symplectic leaf in $P_{2}$. By symmetry, $F \mapsto \rho_{2}\left(\rho_{1}^{-1}(F)\right)$ is a one-to-one correspondence between the symplectic leaves of $P_{1}$ and $P_{2}$. The corresponding leaves have anti-isomorphic normal Poisson structures and, if the manifolds are Morita equivalent, isomorphic first cohomology groups.

2. We1]. The Poisson annihilator of $\rho_{1}^{*} C^{\infty}\left(P_{1}\right)$ in $C^{\infty}(W)$ is $\rho_{2}^{*} C^{\infty}\left(P_{2}\right)$, and vice versa. The manifolds $P_{1}$ and $P_{2}$ have equal spaces of Casimir functions, both isomorphic to $\rho_{1}^{*} C^{\infty}\left(P_{1}\right) \cap \rho_{2}^{*} C^{\infty}\left(P_{2}\right)$.

3. GL. Morita equivalent Poisson manifolds $P_{1}$ and $P_{2}$ have isomorphic first Poisson cohomology spaces. More explicitly, when the fibers of $\rho_{1}$ and $\rho_{2}$ are connected and simply connected, there is a natural isomorphism

$$
E: H_{\pi}^{1}\left(P_{1}\right) \stackrel{\cong}{\rightarrow} H_{\pi}^{1}\left(P_{2}\right) .
$$

We will recall the definition of $E$ in Lemma 5.3 and its proof.

Remark 4.1. In spite of its name, Morita equivalence is not an equivalence relation. However, it becomes such on the class of Poisson manifolds which admit global symplectic groupoids. (See [Xu] for more details.)

4.2. Morita equivalence and the modular class. A Poisson manifold $P$ is said to be locally unimodular if every point of $P$ has a unimodular neighborhood. The key result of this section is the following

Theorem 4.2. Let $P_{1}$ and $P_{2}$ be Morita equivalent and let, in addition, $P_{1}$ be locally unimodular. Then $P_{2}$ is also locally unimodular and $\bmod \left(P_{1}\right)$ goes 
to $\bmod \left(P_{2}\right)$ under the isomorphism $E: H_{\pi}^{1}\left(P_{1}\right) \stackrel{\cong}{\rightarrow} H_{\pi}^{1}\left(P_{2}\right)$ of (9), i. e., $E\left(\bmod \left(P_{1}\right)\right)=\bmod \left(P_{2}\right)$.

In other words, the modular class is an invariant of Morita equivalence of locally unimodular manifolds. The two following particular cases of the theorem deserve a special attention.

Corollary 4.3. A manifold which is Morita equivalent to a unimodular manifold is also unimodular.

Furthermore, since a regular Poisson manifold is automatically locally unimodular, we have

Corollary 4.4. Assume that $P_{1}$ is regular (and, therefore, so is $P_{2}$ ). Then $E\left(\bmod \left(P_{1}\right)\right)=\bmod \left(P_{2}\right)$.

Denote by $H_{*}^{\pi}(P)$ the Poisson homology of $P$, [Br]. When $P$ is unimodular, the pairing with an invariant volume form gives rise to an isomorphism $H_{\pi}^{*}(P) \stackrel{\cong}{\rightrightarrows} H_{m-*}^{\pi}(P)$ with $m=\operatorname{dim} P$, ELW]. Combining Corollary 4.3 with (9), we obtain

Corollary 4.5. Assume that $P_{1}$ is unimodular. Then

$$
H_{m_{1}-1}^{\pi}\left(P_{1}\right) \cong H_{m_{2}-1}^{\pi}\left(P_{2}\right)
$$

where $m_{1}=\operatorname{dim} P_{1}$ and $m_{2}=\operatorname{dim} P_{2}$.

Remark 4.6. In Theorem 4.2, the additional assumption that $P_{1}$ is locally unimodular seems to be purely technical and can probably be removed. Conjecturally, the modular class is an invariant of Morita equivalence for all Poisson manifolds. We also conjecture that the assertion of Corollary 4.5 holds without the requirement that $P_{1}$ be unimodular.

Remark 4.7. As is clear from the proofs, neither the results of [G] nor the results of this section require the dual pair to be complete. It is sufficient to only assume that the fibers of the dual pair are connected and simply connected.

\section{The Proof of TheOrem 4.2}

To explain the idea of the proof, assume first that $P_{1}$ is unimodular. It turns out that then an invariant volume form $\mu_{1}$ on $P_{1}$ gives rise to an invariant form $\mu_{2}$ on $P_{2}$. More precisely, the forms $\rho_{1}^{*} \mu_{1}$ and $\rho_{2}^{*} \mu_{2}$ on $W$ are related by the symplectic $*$-operator, [Br]; see the proof of Lemma 5.2.

To treat the general case, consider the first Chech cohomology $H^{1}(P ; \mathcal{C})$ of a Poisson manifold $P$ with coefficients in the sheaf of Casimir functions $\mathcal{C}$. The cohomology $\check{H}^{1}(P ; \mathcal{C})$ is an invariant of Morita equivalence. There is a natural monomorphism $\Psi_{P}: \check{H}^{1}(P ; \mathcal{C}) \rightarrow H_{\pi}^{1}(P)$. Furthermore, when

\footnotetext{
${ }^{1}$ The isomorphism $E$ used here differs from the one introduced in GL by the sign.
} 
$P$ is locally unimodular, there exists a class $\bmod _{C}(P) \in \check{H}^{1}(P ; \mathcal{C})$, which is $\operatorname{mapped}$ to $\bmod (P)$ by $\Psi_{P}$. The class $\bmod _{C}(P)$ is a global obstruction to the existence of an invariant volume form.

By applying locally the argument we have used for globally unimodular manifolds, we show that $\bmod _{C}(P)$ is an invariant of Morita equivalence. As a consequence, $\bmod (P)=\Psi_{P}\left(\bmod _{C}(P)\right)$ is also an invariant of Morita equivalence.

5.1. Constructions. Let us start with some general remarks on Poisson cohomology. As above, denote by $\mathcal{C}$ the sheaf of Casimir functions on a Poisson manifold $P$. We define the homomorphism

$$
\Psi_{P}: \check{H}^{1}(P ; \mathcal{C}) \rightarrow H_{\pi}^{1}(P)
$$

on the level of cocycles as follows. Pick a cover $\left\{U_{i}\right\}$ of $P$. Let $\left\{f_{i j} \in \mathcal{C}\left(U_{i j}\right)\right\}$, where $U_{i j}=U_{i} \cap U_{j}$, be a one-cocycle of Casimir functions. There exist smooth functions $f_{i}$ on $U_{i}$ such $f_{i j}=f_{i}-f_{j}$. The Hamiltonian vector fields $X_{f_{i}}$ and $X_{f_{j}}$ coincide on the intersections $U_{i j}$ because $f_{i j}$ is Casimir. Hence, there is a Poisson (locally Hamiltonian) vector field $X$ which restricts to $X_{f_{i}}$ on $U_{i}$. By definition, $\Psi_{P}$ sends the cohomology class of $\left\{f_{i j}\right\}$ to the Poisson cohomology class of $X$,

Let now $P_{1} \stackrel{\rho_{1}}{\leftarrow} W \stackrel{\rho_{2}}{\rightarrow} P_{2}$ be a full dual pair with connected and simply connected fibers. Denote by $\mathcal{C}_{1}$ and $\mathcal{C}_{2}$ the sheaves of Casimir functions on $P_{1}$ and, respectively, $P_{2}$.

Lemma 5.1. There is an isomorphism $E_{C}: \check{H}^{1}\left(P_{1} ; \mathcal{C}_{1}\right) \cong \check{H}^{1}\left(P_{2} ; \mathcal{C}_{2}\right)$ associated with this dual pair such that the diagram

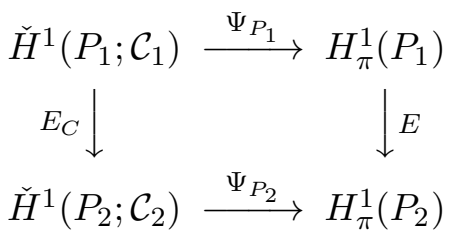

is commutative.

We postpone the proof of Lemma 5.1 and proceed with the proof of the theorem.

Let us incorporate the modular class into the diagram (10). We claim that, when $P$ is locally unimodular, there exists a canonical class $\bmod _{C}(P) \in$ $\check{H}^{1}(P ; \mathcal{C})$ which is mapped to the modular class $\bmod (P)$ by $\Psi_{P}$, i. e.,

$$
\Psi_{P}\left(\bmod _{C}(P)\right)=\bmod (P) .
$$

To construct $\bmod _{C}(P)$, let us cover $P$ by open unimodular neighborhoods $U_{i}$ with invariant volume forms $\mu_{i}$. The ratio $f_{i j}=\mu_{i} / \mu_{j}$ is a smooth function on $U_{i j}=U_{i} \cap U_{j}$. Since $\mu_{i}$ and $\mu_{j}$ are invariant under Hamiltonian flows, $f_{i j}$ is Casimir. Furthermore, $\left\{f_{i j}\right\}$ is a cocycle and, as is easy to see, its cohomology class $\bmod _{C}(P)=\left[\left\{f_{i j}\right\}\right]$ projects to $\bmod (P)$ under $\Psi_{P}$. 
Coming back to Morita equivalent manifolds $P_{1}$ and $P_{2}$, assume that $P_{1}$ is locally unimodular. Since the corresponding symplectic leaves of $P_{1}$ and $P_{2}$ have anti-isomorphic normal Poisson structures, the manifold $P_{2}$ is also locally unimodular. Theorem 4.2 is an immediate consequence of Lemma 5.1 and the following

Lemma 5.2. $E_{C}\left(\bmod _{C}\left(P_{1}\right)\right)=\bmod _{C}\left(P_{2}\right)$.

To complete the proof of the theorem, it remains to prove the lemmas. To this end, let us first characterize the homomorphism $E$ of (9). For a Poisson vector field $\xi$ denote by $[\xi]$ its class in the first Poisson cohomology.

Lemma 5.3. Let $\xi_{1}$ and $\xi_{2}$ be Poisson vector fields on $P_{1}$ and, respectively, $P_{2}$. Then $E\left(\left[\xi_{1}\right]\right)=\left[\xi_{2}\right]$ if and only if there exists a Hamiltonian vector field $\xi$ on $W$ such that

$$
\left(\rho_{1}\right)_{*} \xi=\xi_{1} \quad \text { and } \quad\left(\rho_{2}\right)_{*} \xi=-\xi_{2}
$$

\subsection{Proofs of the lemmas.}

Proof of Lemma 5.3. Let us briefly recall the construction of E. (See GL] for more details.) Observe first that a tangent space to a $\rho_{2}$-fiber is spanned by Hamiltonian vector fields $X_{\rho_{1}^{*} f}$ on $W$, where $f \in C^{\infty}\left(P_{1}\right)$. A Poisson vector field $\xi_{1}$ on $P_{1}$ gives rise to a closed one-form $\alpha_{\xi_{1}}$ along $\rho_{2}$-fibers by the formula

$$
\alpha_{\xi_{1}}\left(X_{\rho_{1}^{*} f}\right)=L_{\xi_{1}} f
$$

Since the $\rho_{2}$-fibers are connected and simply connected, there exists a smooth function $F$ on $W$ such that $\alpha_{\xi_{1}}$ is the restriction of $d F$ to $\rho_{2}$-fibers. Furthermore, as shown in [GL], the push-forward $\xi_{2}=-\left(\rho_{2}\right)_{*} \xi$ of $\xi=X_{f}$ is a well-defined Poisson vector field on $P_{2}$. We set $E\left(\left[\xi_{1}\right]\right)=\left[\xi_{2}\right]$. The function $F$ is defined up to the $\rho_{2}$-pull-back of a smooth function on $P_{2}$, and hence $\left[\xi_{2}\right]$ is independent of the choice of $F$. Moreover, $\left[\xi_{2}\right]$ depends only on the cohomology class $\left[\xi_{1}\right]$, for

$$
\left(\rho_{2}\right)_{*} X_{\rho_{1}^{*} f}=0
$$

for any $f \in C^{\infty}\left(P_{1}\right)$. This shows that the condition of Lemma 5.3 is indeed necessary. Note also that (12) implies that (11) depends only the cohomology classes of $\xi_{1}$ and $\xi_{2}$.

To prove that the condition is sufficient, it is enough to note that, under the hypotheses of Lemma 5.3, $d F=\alpha_{\xi_{1}}$ along $\rho_{2}$-fibers, where $F$ is a Hamiltonian of $\xi$.

Proof of Lemma 5.1. First, let us define the isomorphism $E_{C}$. Denote by $\mathcal{C}=\rho_{1}^{*} \mathcal{C}_{1}$ the pull-back to $W$ of the sheaf $\mathcal{C}_{1}$. There is a natural pull-back homomorphism $\rho_{1}^{*}: \check{H}^{*}\left(P_{1} ; \mathcal{C}_{1}\right) \rightarrow \check{H}^{*}(W ; \mathcal{C})$. Since the fibers of $\rho_{1}$ are connected and simply connected, $\rho_{1}^{*}$ is an isomorphism in the first cohomology. This immediately follows, for example, from the Leray spectral sequence for $\rho_{1}$. (See, e. g., Gode, Section 4.17].) The same argument applies to the 
second projection $\rho_{2}$. On the other hand, $\rho_{2}^{*} \mathcal{C}_{2}=\mathcal{C}=\rho_{1}^{*} \mathcal{C}_{1}$ due to the oneto-one correspondence between symplectic leaves (Property 1 above). Thus, we obtain an isomorphism

$$
E_{C}=\left(\rho_{2}^{*}\right)^{-1} \rho_{1}^{*}: \check{H}^{1}\left(P_{1} ; \mathcal{C}_{1}\right) \rightarrow \check{H}^{1}(W ; \mathcal{C}) \rightarrow \check{H}^{1}\left(P_{2} ; \mathcal{C}_{2}\right) .
$$

Let us show that the diagram (10) is commutative.

First, we will describe the homomorphism $E_{C}$ using cocycles. Fix a cover $\left\{U_{i}\right\}$ of $W$ by small open sets. Let $U_{i}^{\prime}=\rho_{1}\left(U_{i}\right)$ and $U_{i}^{\prime \prime}=\rho_{2}\left(U_{i}\right)$. These are open covers of $P_{1}$ and, respectively, $P_{2}$. For any covers of $W, P_{1}$, and $P_{2}$ one can always find their refinements of the form $\left\{U_{i}\right\},\left\{U_{i}^{\prime}\right\}$, and $\left\{U_{i}^{\prime \prime}\right\}$. Therefore, when working with cohomology, we can use only open covers of this form.

Let $f_{i j}^{\prime}$ and $f_{i j}^{\prime \prime}$ be one-cocycles of Casimir functions on $P_{1}$ and $P_{2}$ with respect to the covers $\left\{U_{i}^{\prime}\right\}$ and, respectively, $\left\{U_{i}^{\prime \prime}\right\}$ such that $\left[f_{i j}^{\prime \prime}\right]=E_{C}\left(\left[f_{i j}^{\prime}\right]\right)$ in the Chech cohomology. This means that, after perhaps taking a refinement of $U_{i}$, we have

$$
\rho_{1}^{*} f_{i j}^{\prime}-\rho_{2}^{*} f_{i j}^{\prime \prime}=\varphi_{i}-\varphi_{j}
$$

on $U_{i j}=U_{i} \cap U_{j}$, where $\varphi_{i} \in \mathcal{C}\left(U_{i}\right)$.

To check the commutativity, we need to prove that

$$
E\left(\Psi_{P_{1}}\left(\left[f_{i j}^{\prime}\right]\right)\right)=\Psi_{P_{2}}\left(\left[f_{i j}^{\prime \prime}\right]\right) .
$$

Let $f_{i}^{\prime}$ and $f_{i}^{\prime \prime}$ be resolutions of $\left\{f_{i j}^{\prime}\right\}$ and, respectively, $\left\{f_{i j}^{\prime \prime}\right\}$ in smooth functions, i. e.,

$$
f_{i j}^{\prime}=f_{i}^{\prime}-f_{j}^{\prime} \quad \text { and } \quad f_{i j}^{\prime \prime}=f_{i}^{\prime \prime}-f_{j}^{\prime \prime}
$$

on their domains. Recall that the Poisson vector fields $\xi_{1}$ and $\xi_{2}$ from the cohomology classes $\Psi_{P_{1}}\left(\left[f_{i j}^{\prime}\right]\right)$ and $\Psi_{P_{2}}\left(\left[f_{i j}^{\prime \prime}\right]\right)$ are locally Hamiltonian vector fields for the families of functions $\left\{f_{i}^{\prime}\right\}$ and $\left\{f_{i}^{\prime \prime}\right\}$, respectively. By Lemma 5.3, to show that the diagram (10) is commutative, i. e., that $E\left(\left[\xi_{1}\right]\right)=\left[\xi_{2}\right]$, it suffices to find a Hamiltonian vector field $\xi$ on $W$ for which (11) holds. Set

$$
F_{i}^{\prime}=\left.\rho_{1}^{*} f_{i}^{\prime}\right|_{U_{i}} \text { and } F_{i}^{\prime \prime}=\left.\rho_{1}^{*} f_{i}^{\prime \prime}\right|_{U_{i}} .
$$

Note that there exists a smooth function $F$ on $W$ such that $\left.F\right|_{U_{i}}=F_{i}^{\prime}-$ $F_{i}^{\prime \prime}-\varphi_{i}$. Indeed, by (13),

$$
\left(F_{i}^{\prime}-F_{i}^{\prime \prime}-\varphi_{i}\right)-\left(F_{j}^{\prime}-F_{j}^{\prime \prime}-\varphi_{j}\right)=0 \quad \text { on } \quad U_{i j} .
$$

Let us show that (11) is satisfied for $\xi=X_{F}$. On $U_{i}$, we have $\left(\rho_{1}\right)_{*} \xi=\left(\rho_{1}\right)_{*}\left(X_{F_{i}^{\prime}}-X_{F_{i}^{\prime \prime}}-X_{\varphi_{i}}\right)=\left(\rho_{1}\right)_{*}\left(X_{\rho_{1}^{*} f_{i}^{\prime}}\right)-\left(\rho_{1}\right)_{*}\left(X_{\rho_{2}^{*} f_{i}^{\prime \prime}}\right)-\left(\rho_{1}\right)_{*}\left(X_{\varphi_{i}}\right)$. Since $\rho_{1}$ is Poisson, the first term is $X_{f_{i}^{\prime}}$, the second term is zero by (12) with $\rho_{1}$ and $\rho_{2}$ interchanged, and the last term is zero because $\varphi_{i} \in \mathcal{C}\left(U_{i}\right)$. This shows that $\left(\rho_{1}\right)_{*} \xi$ is well defined on $U_{i}$ and equal to $\left.\xi_{1}\right|_{U_{i}^{\prime}}$. As a consequence, $\left(\rho_{1}\right)_{*} \xi$ is well defined and equal to $\xi_{1}$ everywhere. For the second submersion $\rho_{2}$, the argument is similar. 
Proof of Lemma 5.2. Assume first that $P_{1}$ is unimodular. To show that $P_{2}$ is unimodular, let us construct a volume form $\mu$ " on $P_{2}$ which is preserved by Hamiltonian flows. To this end, consider the duality operator

$$
D: \Omega^{k}(W) \rightarrow \Omega^{2 n-k}(W),
$$

where $2 n=\operatorname{dim} W$, which is equal to, up to a factor, to the symplectic *-operator, $[\mathrm{Br}]$. The operator $D$ is defined as follows. Recall that $\pi^{\#}: \Omega^{k}(W) \rightarrow \mathcal{X}^{k}(W)$, where $\mathcal{X}^{k}(W)$ is the space of $k$-vector fields, is extended from $\Omega^{1}(W)$ by multiplicativity:

$$
\pi^{\#}\left(\alpha_{1} \wedge \cdots \wedge \alpha_{k}\right)=\pi^{\#}\left(\alpha_{1}\right) \wedge \cdots \wedge \pi^{\#}\left(\alpha_{k}\right),
$$

where $\alpha_{i}$ are one-forms. Recall also that the contraction $\iota_{w} \omega^{n}$ of a $k$-vector field $w$ with the symplectic volume form $\omega^{n}$ is a differential form of degree $2 n-k$. Then, for $\alpha \in \Omega^{k}(W)$, we set $D(\alpha)=\iota_{\pi}{ }_{\alpha} \omega^{n}$.

Let us pick $\mu^{\prime}$, a volume form on $P_{1}$, which is preserved by Hamiltonian flows. We claim that the form $D\left(\rho_{1}^{*} \mu^{\prime}\right)$ on $W$ is the $\rho_{2}$-pull-back of an invariant volume form $\mu^{\prime \prime}$ on $P_{2}$ :

$$
\rho_{2}^{*} \mu^{\prime \prime}=D\left(\rho_{1}^{*} \mu^{\prime}\right) .
$$

Let us first prove that the form $\mu^{\prime \prime}$ exists. Since the $\rho_{2}$-fibers are connected, this will follow if we show that

- $D\left(\rho_{1}^{*} \mu^{\prime}\right)$ is preserved by the Hamiltonian flows on $W$ of functions $\rho_{1}^{*} f$, where $f \in C^{\infty}\left(P_{1}\right)$, and

- $\iota_{v} D\left(\rho_{1}^{*} \mu^{\prime}\right)$ for any $v$ tangent to a $\rho_{2}$-fiber.

The first assertion is equivalent to that $\rho_{1}^{*} \mu^{\prime}$ is invariant, because $\omega^{n}$ and $\pi^{\#}$ are invariant with respect to Hamiltonian flows on $W$. Since $\rho_{1}$ is Poisson, we have

$$
L_{X_{\rho_{1}^{*}}} \rho_{1}^{*} \mu^{\prime}=\rho_{1}^{*}\left(L_{X_{f}} \mu^{\prime}\right) .
$$

By the assumption, $\mu^{\prime}$ is preserved by Hamiltonian flows on $P_{1}$ and so $L_{X_{f}} \mu^{\prime}=0$.

The second assertion is equivalent to that $v \wedge \pi^{\#}\left(\rho_{1}^{*} \mu^{\prime}\right)=0$. Since tangent vectors to $\rho_{2}$-fibers have the form $X_{\rho_{1}^{*} f}$ for $f \in C^{\infty}\left(P_{2}\right)$, we can assume that

$$
v=X_{\rho_{1}^{*} f}=\pi^{\#}\left(d \rho_{1}^{*} f\right) \text {. }
$$

Hence, by the multiplicativity of $\pi^{\#}$, we have

$$
v \wedge \pi^{\#}\left(\rho_{1}^{*} \mu^{\prime}\right)=\pi^{\#}\left(d \rho_{1}^{*} f\right) \wedge \pi^{\#}\left(\rho_{1}^{*} \mu^{\prime \prime}\right)=\pi^{\#}\left(\rho_{1}^{*}\left(d f \wedge \mu^{\prime}\right)\right)=\pi^{\#}\left(\rho_{1}^{*} 0\right)=0 .
$$

Let us prove that $\mu^{\prime \prime}$ is invariant with respect to the Hamiltonian flows. Pick $g \in C^{\infty}\left(P_{2}\right)$. Then $L_{X_{g}} \mu^{\prime \prime}=0$ if and only if $\rho_{2}^{*} L_{X_{g}} \mu^{\prime \prime}=0$. On the other hand,

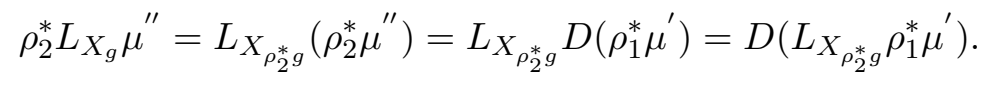

Furthermore,

$$
L_{X_{\rho_{2}^{*} g}}\left(\rho_{1}^{*} \mu^{\prime}\right)=\iota_{X_{\rho_{2}^{*} g}}\left(d \rho_{1}^{*} \mu^{\prime}\right)=0
$$


because $X_{\rho_{2}^{*} g}$ is tangent to the $\rho_{1}$-fibers. This completes the proof of the lemma for unimodular manifolds.

Remark 5.4. The above argument alone is sufficient to prove the theorem in the case where $P_{1}$ is unimodular. Note also that this proof does not require $\rho_{1}$ and $\rho_{2}$ to be complete.

Let us turn to the case of locally unimodular manifolds. In the notation of the proof of Lemma 5.1, choose the cover $\left\{U_{i}\right\}$ so small that all $U_{i}^{\prime}=\rho_{1}\left(U_{i}\right)$ and $U_{i}^{\prime \prime}=\rho_{2}\left(U_{i}\right)$ are unimodular and such that the dual pair $U_{i}^{\prime} \stackrel{\rho_{1}}{\leftarrow} U_{i} \stackrel{\rho_{2}}{\rightarrow} U_{i}^{\prime \prime}$ has connected and simply connected fibers. Fix volume forms $\mu_{i}^{\prime}$ on $U_{i}^{\prime}$ which are preserved by Hamiltonian flows. Denote by $\mu_{i}^{\prime \prime}$ the invariant volume forms on $U_{i}^{\prime \prime}$ such that

$$
\rho_{1}^{*} \mu_{i}^{\prime}=\rho_{2}^{*} \mu_{i}^{\prime \prime}
$$

on $U_{i}$. By definition, the Casimir one-cocycle $f_{i j}^{\prime}=\mu_{i}^{\prime} / \mu_{j}^{\prime}$ represents the class $\bmod _{C}\left(P_{1}\right)$ and $f_{i j}^{\prime \prime}=\mu_{i}^{\prime \prime} / \mu_{j}{ }^{\prime \prime}$ represents $\bmod _{C}\left(P_{2}\right)$. By (14), we have

$$
\rho_{2}^{*} f_{i j}^{\prime \prime}=\rho_{1}^{*} \mu_{i}^{\prime} / \rho_{1}^{*} \mu_{j}^{\prime}=\rho_{1}^{*} f_{i j}^{\prime}
$$

on $U_{i j}$. Therefore $E_{C}\left(\bmod _{C}\left(P_{1}\right)\right)=\bmod _{C}\left(P_{2}\right)$.

This concludes the proof of Theorem 4.2.

Remark 5.5. Let $P$ be a Poisson manifold. Denote by $\mathcal{H}_{P}^{*}$ the sheaf associated with the pre-sheaf of Poisson cohomology, i. e., with the pre-sheaf $U \mapsto H_{\pi}^{*}(U)$, where $U$ is open in $P$ (cf., ELW, Section 6]). Clearly, there is a natural homomorphism $H_{\pi}^{1}(P) \rightarrow \check{H}^{0}\left(\bar{P} ; \mathcal{H}_{P}^{1}\right)$, where $\breve{H}^{0}$ is just the space of sections. The kernel of this homomorphism is exactly the space of the locally Hamiltonian vector fields, i. e., the image of $\Psi_{P}: \check{H}^{1}(P ; \mathcal{C}) \rightarrow H_{\pi}^{1}(P)$. It is easy to see that $\Psi_{P}$ is a monomorphism. Summarizing, we see that the sequence

$$
0 \rightarrow \check{H}^{1}(P ; \mathcal{C}) \rightarrow H_{\pi}^{1}(P) \rightarrow \check{H}^{0}\left(P ; \mathcal{H}_{P}^{1}\right)
$$

is exact (cf., [BZ]). The image of $\bmod (P)$ in $\check{H}^{0}\left(P ; \mathcal{H}_{P}^{1}\right)$ is the local obstruction to the existence of an invariant volume form. If this obstruction vanishes, there exists a "global" obstruction $\bmod _{C}(P) \in \check{H}^{1}(P ; \mathcal{C})$.

Furthermore, let $P_{1}$ and $P_{2}$ be Morita equivalent. Then, in the notation of the proof of Theorem 4.2, we obtain the following commutative diagram:

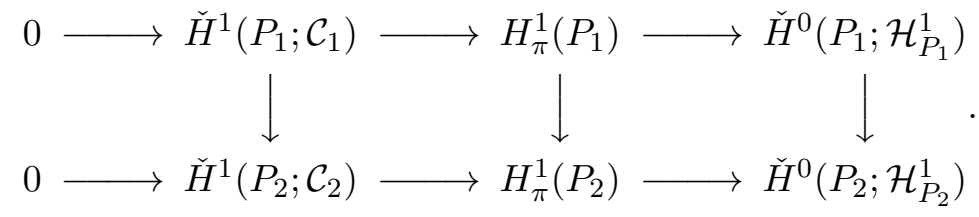

The commutativity of the left square is the assertion of Lemma 5.1. The existence of the isomorphism $\check{H}^{0}\left(P_{1} ; \mathcal{H}_{P_{1}}^{1}\right) \rightarrow \check{H}^{0}\left(P_{2} ; \mathcal{H}_{P_{2}}^{1}\right)$ follows from the fact, [We1], that the corresponding leaves of $P_{1}$ and $P_{2}$ have anti-isomorphic 
normal Poisson structures. The commutativity of right square can then be checked by a direct calculation.

Remark 5.6. The definition of Morita equivalence of regular foliations mimics in the obvious way the definition for Poisson structures: the pull-backs of foliations by $\rho_{1}$ and $\rho_{2}$ coincide with each other and the $\rho_{1^{-}}$and $\rho_{2}$-fibers are connected and simply connected. Denote by $\mathcal{C}$ the sheaf of functions constant on the leaves of a foliated manifold $P$. Similarly to Lemma 5.1, $\check{H}^{1}(P ; \mathcal{C})$ is an invariant of the Morita equivalence of foliations. Moreover, the same is true for $\check{H}^{j}(P ; \mathcal{C}), j \leq k$, when the fibers are assumed to be $k$ connected. It is easy to see that the Godbillon-Vey class of codimension-one foliations is an invariant of Morita equivalence with three-connected fibers.

\section{REFERENCES}

[Br] Brylinski, J.-L., A differential complex for Poisson manifolds, J. Differential Geom., 28 (1988), 93-114.

[BZ] Brylinski, J.-L., Zuckerman, G., The outer derivation of a complex Poisson manifold, Preprint 1997, math.DG/9802014.

[CW] Cannas da Silva, A., Weinstein, A., Lectures on geometric models for noncommutative algebras, to be published in the Berkeley Mathematics Lecture Notes series; available at http://math.berkeley.edu/ alanw/.

[Co] Connes, A., Noncommutative geometry, Academic Press, San Diego, 1994.

[Da1] Dazord, P. Holonomie des feuilletages singuliers, C.R. Acad. Sci. Paris, 298 (1984), 27-30.

[Da2] Dazord, P., Feuilletages à singularités, Nederl. Akad. Wetensch. Indag. Math., 47 (1985), 21-39.

[ELW] Evens, S., Lu, J.-H., Weinstein, A., Transverse measures, the modular class, and a cohomology pairing for Lie algebroids, Preprint math.DG/9610108, to appear in Quart. J. Math..

[GL] Ginzburg, V. L., Lu, J.-H., Poisson cohomology of Morita equivalent Poisson manifolds, IMRN, 10 (1992), 199-205.

[Godb] Godbillon, C., Feuilletages. Études géométriques, Progress in Math. 98, Birkhauser, Boston, 1991.

[Gode] Godement, R., Topologie algébrique et théorie des faisceaux, Hermann, Paris, 1958.

[Ko] Koszul, J. L., Crochet de Schouten-Nijenhuis et cohomologie, Astérisque, hors serie, Soc. Math. France, Paris (1985), 257-271.

[SM] Siegel, C. L., Moser, J. K., Lectures on celestial mechanics, Springer-Verlag, New York, 1971.

[Va] Vaisman, I., Lectures on the geometry of Poisson manifolds, Progress in Math. 118, Birkhauser, Boston, 1994.

[We1] Weinstein, A., The local structure of Poisson manifolds, J. Differential Geom., 18 (1983), 523-557.

[We2] Weinstein, A., Lagrangian mechanics and groupoids, Mechanics day (Waterloo, ON, 1992), 207-231, Fields Inst. Commun., 7, Amer. Math. Soc., Providence, RI, 1996.

[We3] Weinstein, A., The modular automorphism group of a Poisson manifold, J. Geom. Phys., 23 (1997), 379-394.

[Xu $\mathrm{Xu}, \mathrm{P} ., \mathrm{M}$ Mrita equivalence of Poisson manifolds, Comm. Math. Phys., 142 (1991), 493-509. 
Department of Mathematics, UC Santa Cruz, Santa Cruz, CA 95064, USA

E-mail address: ginzburg@math.ucsc.edu

E-mail address: alexgol@cats.ucsc.edu 